\title{
Measurement of the inclusive production of neutral pions and charged particles on the $Z^{0}$ resonance
}

\author{
L3 Collaboration
}

B. Adeva a , O. Adriani ${ }^{\text {b }}$, M. Aguilar-Benitez ${ }^{\text {c }}$, H. Akbari ${ }^{\text {d }}$, J. Alcaraz $^{c}$, A. Aloisio ${ }^{\text {e }}$, G. Alverson ${ }^{\mathrm{f}}$, M.G. Alviggi ${ }^{\mathrm{e}}$, Q. An ${ }^{\mathrm{g}}$, H. Anderhub ${ }^{\mathrm{h}}$, A.L. Anderson ${ }^{\mathrm{i}}$, V.P. Andreev ${ }^{\mathrm{j}}$, T. Angelov i, L. Antonov ${ }^{\text {k }}$, D. Antreasyan ${ }^{\ell}$, P. Arce ${ }^{\text {c }}$, A. Arefiev ${ }^{\text {m }}$, T. Azemoon ${ }^{\text {n }}$, T. Aziz ${ }^{\circ}$, P.V.K.S. Baba ${ }^{\text {g }}$, P. Bagnaia ${ }^{\text {p }}$, J.A. Bakken ${ }^{\mathrm{q}}$, L. Baksay ${ }^{\mathrm{r}}$, R.C. Ball ${ }^{\mathrm{n}}$, S. Banerjee ${ }^{\circ}$, J. Bao $^{\text {d, }}$, L. Barone $^{\text {p }}$, A. Bay ${ }^{\text {s }}$, U. Becker ${ }^{\text {i }}$, J. Behrens ${ }^{\text {h }}$, S. Beingessner ${ }^{\text {t }}$, Gy.L. Bencze ${ }^{\text {u,a }}$, J. Berdugo ${ }^{\text {c }}$, P. Berges ${ }^{\mathrm{i}}$, B. Bertucci ${ }^{\mathrm{p}}$, B.L. Betev ${ }^{\mathrm{k}}$, A. Biland ${ }^{\mathrm{h}}$, R. Bizzarri ${ }^{\mathrm{p}}$, J.J. Blaising ${ }^{\mathrm{t}}$, P. Blömeke ${ }^{\mathrm{v}}$, B. Blumenfeld ${ }^{\mathrm{d}}$, G.J. Bobbink ${ }^{\mathrm{w}}$, M. Bocciolini ${ }^{\mathrm{b}}$, R. Bock ${ }^{\mathrm{v}}$, A. Böhm ${ }^{\mathrm{v}, \mathrm{a}}$, B. Borgia ${ }^{\mathrm{p}}$, D. Bourilkov ${ }^{k}$, M. Bourquin ${ }^{\text {s }}$, D. Boutigny ${ }^{\mathrm{t}}$, B. Bouwens ${ }^{\text {w }}$, J.G. Branson ${ }^{\mathrm{x}}$, I.C. Brock ${ }^{\mathrm{y}}$, F. Bruyant ${ }^{\text {a }}$, C. Buisson ${ }^{\text {z }}$, A. Bujak ${ }^{\text {aa }}$, J.D. Burger ${ }^{\text {, }}$, J.P. Burq ${ }^{z}$, J. Busenitz ${ }^{\mathrm{r}}$, X.D. Cai ${ }^{\mathrm{g}}$, M. Capell ${ }^{\text {ab }}$, F. Carbonara ${ }^{\text {e }}$, P. Cardenal ${ }^{\text {a }}$, F. Carminati ${ }^{\text {b }}$, A.M. Cartacci ${ }^{\text {b }}$, M. Cerrada ${ }^{\text {c }}$, F. Cesaroni ${ }^{\text {p }}$, Y.H. Chang ${ }^{\mathrm{i}}$, U.K. Chaturvedi ${ }^{\mathrm{g}}$, M. Chemarin ${ }^{\mathrm{z}}$, A. Chen ${ }^{\mathrm{ac}}$, C. Chen ${ }^{\text {ad }}$,

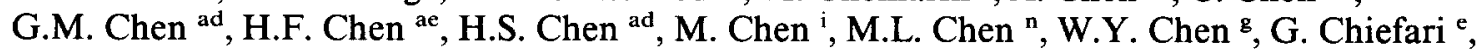
C.Y. Chien ${ }^{\text {d }, ~ F . ~ C h o l l e t ~}{ }^{\mathrm{t}}$, C. Civinini ${ }^{\mathrm{b}}$, I. Clare ${ }^{\mathrm{i}}$, R. Clare ${ }^{\mathrm{i}}$, H.O. Cohn ${ }^{\text {af }}$, G. Coignet ${ }^{\mathrm{t}}$, N. Colino ${ }^{a}$, V. Commichau ${ }^{\text {v }}$, G. Conforto ${ }^{b}$, A. Contin ${ }^{a}$, F. Crijns ${ }^{w}$, X.Y. Cui ${ }^{8}$, T.S. Dai ${ }^{\text {i }}$ R. D’Alessandro ${ }^{b}$, R. de Asmundis ${ }^{e}, A$. Degré ${ }^{a, t}, K$. Deiters ${ }^{i}$, E. Dénes ${ }^{\text {u,a }}$, P. Denes ${ }^{\mathrm{q}}$, F. DeNotaristefani ${ }^{p}$, M. Dhina $^{h}$, D. DiBitonto ${ }^{r}$, M. Diemoz ${ }^{p}$, F. Diez-Hedo ${ }^{a}$, H.R. Dimitrov ${ }^{k}$, C. Dionisi ${ }^{\mathrm{p}}$, R. Divià ${ }^{\mathrm{a}}$, M.T. Dova ${ }^{\mathrm{g}}$, E. Drago ${ }^{\mathrm{e}}$, T. Driever ${ }^{\mathrm{w}}$, D. Duchesneau s, P. Duinker w, I. Duran ${ }^{\text {c }}$, H. El Mamouni ${ }^{z}$, A. Engler ${ }^{\text {y }}$, F.J. Eppling ${ }^{\text {i }}$, F.C. Erné ${ }^{w}$, P. Extermann ${ }^{\text {s }}$, R. Fabbretti ${ }^{\text {ag }}$, G. Faber $^{\text {h }}$, M. Fabre ${ }^{\text {h }}$, S. Falciano ${ }^{\text {p }}$, Q. Fan ${ }^{\mathrm{g}}$, S.J. Fan ${ }^{\text {ah }}$, O. Fackler ${ }^{\text {ab }}$, J. Fay ${ }^{\text {, }}$, J. Fehlmann ${ }^{\text {h }}$, T. Ferguson ${ }^{\text {y }}$, G. Fernandez ${ }^{\text {c }}$, F. Ferroni ${ }^{\text {pa }}$, H. Fesefeldt ${ }^{v}$, J. Field s , F. Filthaut ${ }^{\text {w }}$, G. Finocchiaro ${ }^{\text {p }, ~ P . H . ~ F i s h e r ~}{ }^{\text {d }}$, G. Forconi ${ }^{\text {s, }}$ T. Foreman "w K. Freudenreich ${ }^{\text {h }}$, W. Friebel ai, M. Fukushima ${ }^{\mathrm{i}}$, M. Gailloud aj, Yu. Galaktionov ${ }^{m}$, E. Gallo ${ }^{\text {b }, ~ S . N . ~ G a n g u l i ~}{ }^{\circ}$, P. Garcia-Abia ${ }^{c}$, S.S. Gau ${ }^{\text {ac }}$, D. Gele ${ }^{\text {, }}$ S. Gentile ${ }^{p}$, M. Glaubman ${ }^{\text {f }}$, S. Goldfarb ${ }^{n}$, Z.F. Gong ${ }^{\text {ae }}$, E. Gonzalez ${ }^{c}$, A. Gordeev ${ }^{\mathrm{m}}$, P. Göttlicher ${ }^{v}$, D. Goujon ${ }^{\text {s }}$, G. Gratta ${ }^{\text {ak }}$, C. Grinnell ${ }^{i}$, M. Gruenewald ${ }^{\text {ak }}$, M. Guanziroli ${ }^{\mathrm{g}}$, J.K. Guo ah , A. Gurtu ${ }^{\text {o, H.R. Gustafson }}{ }^{\text {n }}$, L.J. Gutay ${ }^{\text {aa }}$, H. Haan ${ }^{\text {v }}$, A. Hasan ${ }^{\text {g, }}$, D. Hauschildt ${ }^{\text {w }}$, C.F. He ${ }^{\text {ah }}$, T. Hebbeker ${ }^{v}$, M. Hebert ${ }^{\text {x }}$, G. Herten ${ }^{i}$, U. Herten ${ }^{\text {v }}$, A. Hervé a, K. Hilgers ${ }^{\text {v }}$, H. Hofer ${ }^{\text {h }}$, H. Hoorani ${ }^{\text {g }}$, L.S. Hsu ${ }^{\text {ac }}$, G. Hu ${ }^{\text {g }}$, G.Q. Hu ${ }^{\text {ah }}$, B. Ille ${ }^{\text {z }}$, M.M. Ilyas ${ }^{\text {g }}$, V. Innocente ${ }^{\text {e.a }}$, E. Isiksal ${ }^{\text {h }}$, H. Janssen ${ }^{\text {a }}$, B.N. Jin ${ }^{\text {ad }}$, L.W. Jones ${ }^{\text {n }}$, A. Kasser ${ }^{\text {aj }}$, R.A. Khan ${ }^{8}$, Yu. Kamyshkov ${ }^{\text {m,af }}$, Y. Karyotakis ${ }^{\text {t,a }}$, M. Kaur ${ }^{\text {g }}$, S. Khokhar ${ }^{\text {g }}$, V. Khoze ${ }^{\mathrm{j}}$, M.N. Kienzle-Focacci ${ }^{\text {s }}$, W. Kinnison ${ }^{\text {a }}$, D. Kirkby ${ }^{\text {ak }}$, W. Kittel ${ }^{\text {w }}$, A. Klimentov ${ }^{m}$, A.C. König " , O. Kornadt ${ }^{\text {, }, ~ V . ~ K o u t s e n k o ~}{ }^{\text {m.1 }}$, R.W. Kraemer ${ }^{y}$, T. Kramer ${ }^{\text {, }}$, V.R. Krastev ${ }^{\text {, }}$, W. Krenz ${ }^{\text {v }}$, J. Krizmanic ${ }^{\text {d, K.S. Kumar }}{ }^{\text {am }}$, V. Kumar ${ }^{\text {g }}$, A. Kunin ${ }^{\text {m }}$, V. Lalieu ${ }^{\text {s, G. Landi }}{ }^{\text {, }}$, D. Lanske ${ }^{\text {v }}$, S. Lanzano ${ }^{\text {e }}$, P. Lebrun ${ }^{\text {, }}$, P. Lecomte ${ }^{\mathrm{h}}$, P. Lecoq ${ }^{\mathrm{a}}$, P. Le Coultre ${ }^{\mathrm{h}}$, D. Lee ${ }^{\mathrm{al}}$, I. Leedom ${ }^{\text {f }}$, J.M. Le Goff ${ }^{a}$, L. Leistam ${ }^{a}$, R. Leiste ${ }^{\text {ai }}$, M. Lenti ${ }^{b}$, E. Leonardi ${ }^{p}$, J. Lettry $^{\text {h }}$,

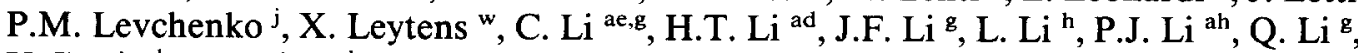
X.G. Li ${ }^{\text {ad }}$, J.Y. Liao ${ }^{\text {ah }}$, Z.Y. Lin ${ }^{\text {ae }}$, F.L. Linde ${ }^{y}$, B. Lindemann ${ }^{\text {v }, ~ D . ~ L i n n h o f e r ~}{ }^{\mathrm{h}}$, R. Liu ${ }^{\mathrm{g}}$,

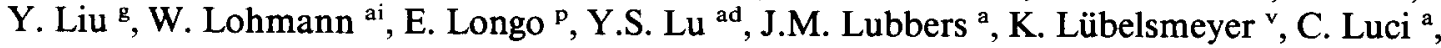




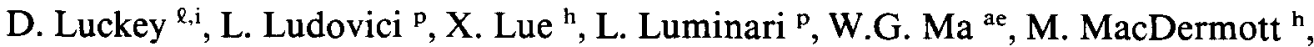
R. Magahiz an ${ }^{\text {an }}$ M. Maire ${ }^{\text {t }}$, P.K. Malhotra ${ }^{\circ}$, R. Malik ${ }^{\mathrm{g}}$, A. Malinin ${ }^{\mathrm{m}}$, C. Maña ${ }^{\mathrm{c}}$, D.N. Mao ${ }^{\mathrm{n}}$, Y.F. Mao ${ }^{\text {ad }}$, M. Maolinbay ${ }^{\text {h }}$, P. Marchesini ${ }^{\text {h }}$, A. Marchionni ${ }^{\text {b }}$, B. Martin ${ }^{\text {a }}$, J.P. Martin ${ }^{\text {, }}$, L. Martinez-Laso a , F. Marzano ${ }^{\text {p }}$, G.G.G. Massaro ${ }^{\text {w }}$, T. Matsuda ${ }^{\text {i }}$, K. Mazumdar ${ }^{\text {o, }}$ P. McBride ${ }^{\text {am }}$, T. McMahon ${ }^{\text {aa }}$, D. McNally ${ }^{\text {h }}$, Th. Meinholz ${ }^{\mathrm{v}}$, M. Merk ${ }^{\text {w }}$, L. Merola ${ }^{\mathrm{e}}$, M. Meschini ${ }^{\text {b }}$, W.J. Metzger ${ }^{\text {w }}$, Y. Mi ${ }^{\mathrm{g}}$, G.B. Mills ${ }^{\mathrm{al}}$, Y. Mir ${ }^{\mathrm{g}}$, G. Mirabelli ${ }^{\mathrm{p}}$, J. Mnich ${ }^{\mathrm{v}}$, M. Möller ${ }^{\text {v }}$, B. Monteleoni ${ }^{\mathrm{b}}$, G. Morand ${ }^{\mathrm{s}}$, R. Morand ', S. Morganti ${ }^{\mathrm{p}}$, N.E. Moulai ${ }^{\mathrm{g}}$, R. Mount ${ }^{\text {ak }}$, S. Müller ${ }^{\text {v }, ~ E . ~ N a g y ~}{ }^{\text {u }}$, M. Napolitano ${ }^{\mathrm{e}}$, H. Newman ${ }^{\text {ak }}$, C. Neyer ${ }^{\text {h }}$, M.A. Niaz ${ }^{\mathrm{g}}$, L. Niessen ${ }^{\text {v }}$, H. Nowak ai, D. Pandoulas ${ }^{\text {v }, ~ F . ~ P l a s i l ~ a f, ~ G . ~ P a s s a l e v a ~}{ }^{\text {b }}$, G. Paternoster ${ }^{\text {e, }}$ S. Patricelli e , Y.J. Pei v, D. Perret-Gallix ${ }^{\mathrm{t}}$, J. Perrier ${ }^{\mathrm{s}}$, A. Pevsner ${ }^{\mathrm{d}}$, M. Pieri ${ }^{\mathrm{b}}$, P.A. Piroué ${ }^{\mathrm{q}}$, V. Plyaskin ${ }^{\mathrm{m}}$, M. Pohl ${ }^{\mathrm{h}}$, V. Pojidaev ${ }^{\mathrm{m}}$, N. Produit ${ }^{\mathrm{s}}$, J.M. Qian ${ }^{\mathrm{n}}$, K.N. Qureshi ${ }^{\mathrm{s}}$, R. Raghavan ${ }^{\circ}$, G. Rahal-Callot ${ }^{\mathrm{h}}$, P. Razis ${ }^{\mathrm{r}}, \mathrm{K}$. Read $^{\text {af }}, \mathrm{D}$. Ren ${ }^{\mathrm{h}}$, Z. Ren ${ }^{\mathrm{g}}, \mathrm{S}_{\text {. Reucroft }}{ }^{\mathrm{f}}$, A. Ricker ${ }^{\text {v }}$ S. Riemann ${ }^{\text {ai }}$, O. Rind ${ }^{\text {n, C. Rippich }}{ }^{y}$, H.A. Rizvi ${ }^{\mathrm{g}}$, B.P. Roe ${ }^{\mathrm{n}}$, M. Röhner ${ }^{\mathrm{v}}$, S. Röhner ${ }^{\text {v }}$, U. Roeser ${ }^{\text {h }}$, L. Romero ${ }^{\text {c }}$, J. Rose ${ }^{\text {v }}$, S. Rosier-Lees ${ }^{\text {t }}$, R. Rosmalen ${ }^{\text {w, }}$ Ph. Rosselet aj, A. Rubbia ${ }^{\text {i }, ~ J . A . ~ R u b i o ~}{ }^{\text {a.c }}$, M. Rubio ${ }^{\text {a }}$, W. Ruckstuhl ${ }^{\text {s }}$, H. Rykaczewski ${ }^{\text {h }}$, M. Sachwitz ai.a , J. Salicio ${ }^{\text {a.c }}$, J.M. Salicio c ${ }^{\text {, G. Sanders }}{ }^{\text {a }}$, M.S. Sarakinos ${ }^{i}$, G. Sartorelli ${ }^{\ell, z}$, G. Sauvage ${ }^{t}$, A. Savin ${ }^{m}$, V. Schegelsky ${ }^{j}$, K. Schmiemann ${ }^{\text {v }}$, D. Schmitz v , P. Schmitz v, M. Schneegans ', H. Schopper ${ }^{\text {ao }}$, D.J. Schotanus ${ }^{\text {w }}$, S. Shotkin ${ }^{\text {i }}$, H.J. Schreiber ${ }^{\text {ai }}$, R. Schulte ${ }^{\text {, }}$, S. Schulte v, K. Schultze v, J. Schütte ${ }^{\mathrm{am}}$, J. Schwenke v , G. Schwering v , C. Sciacca ${ }^{\text {e I. Scott }}{ }^{\text {am }}$, R. Sehgal ${ }^{\text {g }}$, P.G. Seiler ${ }^{\text {ag }}$, J.C. Sens ${ }^{\text {w }}$, I. Sheer ${ }^{x}$, D.Z. Shen ${ }^{\text {ah }}$, V. Shevchenko ${ }^{m}$,

S. Shevchenko ${ }^{m}$, X.R. Shi ${ }^{y}$, K. Shmakov ${ }^{m}$, V. Shoutko ${ }^{m}$, E. Shumilov ${ }^{m}$, N. Smirnov ${ }^{j}$, E. Soderstrom ${ }^{\text {a }}$, A. Sopczak ${ }^{x}$, C. Spartiotis ${ }^{\text {d, }}$ T. Spickermann ${ }^{\text {v }}$, B. Spiess ${ }^{\text {h }}$, P. Spillantini ${ }^{b}$, R. Starosta ${ }^{v}$, M. Steuer ${ }^{\text {l,i }}$, D.P. Stickland ${ }^{\text {q }}$, F. Sticozzi ${ }^{i}$, W. Stoeffl ${ }^{\text {ab }}$, H. Stone ${ }^{\text {, }}$, K. Strauch ${ }^{\text {am }}$, B.C. Stringfellow ${ }^{\text {aa }}$, K. Sudhakar ${ }^{\text {o,v }}$, G. Sultanov ${ }^{\text {g }}$, R.L. Sumner ${ }^{\text {q }}$, L.Z. Sun ${ }^{\text {ae,g, }}$, H. Suter ${ }^{\text {h }}$, R.B. Sutton y , J.D. Swain ${ }^{\text {g }}$, A.A. Syed ${ }^{\text {g }}$, X.W. Tang ${ }^{\text {ad }}$, E. Tarkovsky ${ }^{\mathrm{m}}$, L. Taylor ${ }^{\mathrm{f}}$, C. Timmermans ${ }^{\text {w }}$, Samuel C.C. Ting ${ }^{\text {i }}$, S.M. Ting ${ }^{\text {i }}$, Y.P. Tong ${ }^{\text {ac }}$, F. Tonisch ${ }^{\text {ai }}$, M. Tonutti ${ }^{\text {, }}$, S.C. Tonwar ${ }^{\circ}$, J. Tóth ${ }^{\text {u.a }}$, G. Trowitzsch ${ }^{\text {ai }}$, C. Tully ${ }^{\text {ak }}$, K.L. Tung ${ }^{\text {ad }}$, J. Ulbricht ${ }^{\mathrm{h}}$, L. Urbán ${ }^{\mathrm{u}}$, U. Uwer $^{\mathrm{v}}$, E. Valente ${ }^{\mathrm{p}}$, R.T. Van de Walle ${ }^{\mathrm{w}}$, I. Vetlitsky ${ }^{\mathrm{m}}$, G. Viertel $^{\mathrm{h}}$, P. Vikas ${ }^{\mathrm{g}}$, U. Vikas ${ }^{\mathrm{g}}$, M. Vivargent ${ }^{t, i}$, H. Vogel ${ }^{y}, H$. Vogt ${ }^{\text {ai }}$, G. Von Dardel ${ }^{a}$, I. Vorobiev ${ }^{m}$, A.A. Vorobyov ${ }^{j}$, An.A. Vorobyov ${ }^{j}$, L. Vuilleumier ${ }^{\text {aj }}$, M. Wadhwa ${ }^{g}$, W. Wallraff ${ }^{\mathrm{v}}$, C.R. Wang ${ }^{\text {ae }}$, G.H. Wang ${ }^{\mathrm{y}}$, J.H. Wang ${ }^{\text {ad }}$, Q.F. Wang ${ }^{\text {am }}$, X.L. Wang ${ }^{\text {ae }}$, Y.F. Wang ${ }^{\mathrm{b}}$, Z. Wang ${ }^{\mathrm{g}}$, Z.M. Wang ${ }^{\mathrm{g}, a e}$, A. Weber ${ }^{\mathrm{v}}$, J. Weber ${ }^{\text {h }}$, R. Weill aj, T.J. Wenaus ${ }^{\text {ab }}$, J. Wenninger ${ }^{\text {s }}$, M. White ${ }^{i}$, C. Willmott $^{c}$,

F. Wittgenstein ${ }^{\text {a }}$, D. Wright ${ }^{\mathrm{a}}$, R.J. Wu ${ }^{\text {ad }}$, S.L. Wu ${ }^{\text {g }}$, S.X. Wu ${ }^{\mathrm{g}}$, Y.G. Wu ${ }^{\text {ad }}$, B. Wysłouch ${ }^{\mathrm{i}}$, Y.Y. Xie ${ }^{\text {ah }}$, Y.D. Xu ${ }^{\text {ad }}$, Z.Z. Xu ${ }^{\text {ae }}$, Z.L. Xue ${ }^{\text {ah }}$, D.S. Yan ${ }^{\text {ah }}$, X.J. Yan ${ }^{\text {i }, ~ B . Z . ~ Y a n g ~}{ }^{\text {ae }}$, C.G. Yang ad, G. Yang ${ }^{\text {g }}$, K.S. Yang ${ }^{\text {ad }}$, Q.Y. Yang ${ }^{\text {ad }}$, Z.Q. Yang ${ }^{\text {ah }}$, C.H. Ye ${ }^{\text {g }}$, J.B. Ye ${ }^{\text {h,ae }}$, Q. Ye ${ }^{\text {g }, ~ S . C . ~ Y e h ~}{ }^{\text {ac }}$, Z.W. Yin ${ }^{\text {ah }}$, J.M. You ${ }^{\mathrm{g}}$, M. Yzerman ${ }^{\text {w }}$, C. Zaccardelli ${ }^{\text {ak }}$, L. Zehnder ${ }^{\text {h }}$, P. Zemp ${ }^{\text {h }}$, M. Zeng ${ }^{\text {g, Y. Y. Zeng }}{ }^{\text {v }, ~ D . H . ~ Z h a n g ~}{ }^{\text {w }}$, Z.P. Zhang ${ }^{\text {ae, }, ~ J . F . ~ Z h o u ~}{ }^{\text {v }}$, R.Y. Zhu ${ }^{\text {ak }}$, H.L. Zhuang ad and A. Zichichi ${ }^{\text {a,g }}$

a European Laboratory for Particle Physics, CERN, CH-1211 Geneva 23, Switzerland

- INFN - Sezione di Firenze and University of Firenze, I-50125 Florence, Italy

c Centro de Investigaciones Energeticas, Medioambientales y Tecnologicas, CIEMAT, E-28040 Madrid, Spain

d Johns Hopkins University, Baltimore, MD 21218, USA

- INFN - Sezione di Napoli and University of Naples, I-80125 Naples, Italy

f Northeastern University, Boston, MA 02115, USA

g World Laboratory, FBLJA Project, CH-1211 Geneva, Switzerland

h Eidgenössische Technische Hochschule, ETH Zürich, CH-8093 Zurich, Switzerland Massachusetts Institute of Technology, Cambridge, MA 02139, USA

Leningrad Nuclear Physics Institute, SU-188 350 Gatchina, USSR

- Central Laboratory of Automation and Instrumentation, CLANP, Sofia, Bulgaria 
I INFN - Sezione di Bologna, 1-40126 Bologna, Italy

m Institute of Theoretical and Experimental Physics, ITEP, SU-117259 Moscow, USSR

" University of Michigan, Ann Arbor, MI 48109, USA

- Tata Institute of Fundamental Research, Bombay 400 005, India

" INFN - Sezione di Roma and University of Rome "La Sapienza", I-00185 Rome, Italy

- Princeton University, Princeton, NJ 08544, USA

r University of Alabama, Tuscaloosa, AL 35486, USA

s University of Geneva, CH-1211 Geneva 4, Switzerland

- Laboratoire de Physique des Particules, LAPP, F-74519 Annecy-le-Vieux, France

" Central Research Institute for Physics of the Hungarian Academy of Sciences, H-1525 Budapest 114, Hungary

v I. Physikalisches Institut, RWTH, W-5100 Aachen, FRG ${ }^{1}$ and III. Physikalisches Institut, RWTH, W-5100 Aachen, FRG

" National Institute for High Energy Physics, NIKHEF, NL-1009 DB Amsterdam, The Netherlands and NIKHEF-H and University of Nijmegen, NL-6525 ED Nijmegen, The Netherlands

* University of California, San Diego, CA 92182, USA

y Carnegie Mellon University, Pittsburgh, PA 15213, USA

2 Institut de Physique Nucléaire de Lyon, IN2P3-CNRS/Université Claude Bernard, F-69622 Villeurbanne Cedex, France :e

aa Purdue University, West Lafayette, IN 47907, USA

ab Lawrence Livermore National Laboratory, Livermore, CA 94550, USA

ac High Energy Physics Group, Taiwan, ROC

ad Institute of High Energy Physics, IHEP, Beijing, China

ac University of Science and Technology of China, Hefei, Anhui 230 029, China

af Oak Ridge National Laboratory, Oak Ridge. TN 37830, USA

as Paul Scherrer Institut (PSI), Würenlingen, Switzerland

ah Shanghai Institute of Ceramics, SIC, Shanghai, China

ai High Energy Physics Institute, O-1615 Zeuthen-Berlin, $F R G^{1}$

aj University of Lausanne, CH-1015 Lausanne, Switzerland

${ }^{\text {ak }}$ California Institute of Technology, Pasadena, CA 91125, USA

al Los Alamos National Laboratory, Los Alamos, NM 87544, USA

am Harvard University, Cambridge, MA 02139, USA

an Union College, Schenectady, NY 12308, USA

ao University of Hamburg, W-2000 Hamburg, FRG

Received 4 February 1991

\begin{abstract}
We present a study of the inclusive production of neutral pions and charged particles from 112000 hadronic $Z^{\circ}$ decays. The measured inclusive momentum distributions can be reproduced by parton shower Monte Carlo programs and also by an analytical QCD calculation. Comparing our results to $\mathrm{e}^{+} \mathrm{e}^{-}$data between $\sqrt{s}=9$ and $91 \mathrm{GeV}$, we find that the evolution of the spectra with center of mass energy is consistent with the QCD predictions.
\end{abstract}

\section{Introduction}

We report here on measurements of inclusive particle production in hadronic events at the $Z^{0}$ resonance using the $\mathrm{L} 3$ detector at LEP. Neutral pions are reconstructed from photon pairs measured in the electromagnetic calorimeter with an invariant mass close to $m_{\pi 0}$. Charged particles are reconstructed us-

Supported by the German Bundesministerium für Forschung und Technologie. ing the central tracking chamber. We determine the spectra as a function of the variables $x_{p}$ and $\ln (1 /$ $x_{p}$ ), where $x_{p}$ denotes the ratio of particle momentum $p$ to the beam energy $\frac{1}{2} \sqrt{s}$. We compare the in clusive distributions for $\pi^{0}$ and charged particles to the predictions of perturbative QCD. In this comparison we include measurements obtained by several other $\mathrm{e}^{+} \mathrm{e}^{-}$experiments at center of mass energies from 9 to $91 \mathrm{GeV}[1-7]$.

We consider two approaches to calculate the inclusive momentum spectra within perturbative QCD: 
(1) Monte Carlo parton shower generators based on leading log calculations including gluon coherence. Particle mass effects are taken into account. Hadronization and decays are simulated. The parameters of the fragmentation models are obtained from a comparison between measured and simulated event shape variables. We use the programs JETSET 7.2 [8] and HERWIG 4.2 [9].

(2) Analytical calculations in "Modified Leading Log Approximation" (MLLA), summing double and single leading-log contributions, and including coherence effects [10]. We use the "limiting spectrum", for which particle mass effects are not included. It is assumed that the calculated parton spectra can be compared directly to the momentum distribution of measured hadrons ("Local Parton Hadron Duality") [10,11].

The most striking prediction of perturbative QCD concerning the inclusive momentum spectra [12] is a reduction of the number of soft gluons due to destructive interference [13]. This behaviour can be studied best in terms of the variable $\xi_{p}=\ln \left(1 / x_{p}\right)$. The QCD calculations predict a maximum in the $\xi_{p}$ distribution $[10,14,15]$. The position of the maximum, $\xi_{p}^{*}$, is expected at $\approx 3.8$ for $\sqrt{s}=91 \mathrm{GeV}$, which corresponds to $x_{p} \approx 0.02$ and $p \approx 1 \mathrm{GeV}$. At higher values of $\xi_{p}$ the cross sections are reduced due to soft gluon coherence. The position of the maximum $\xi_{p}^{*}$ is expected to move to higher values with increasing center of mass energy. For massive particles the limiting spectrum is modified such that the peak position is shifted to lower values. Thus one expects that for charged particles, which include, in addition to pions, heavier hadrons, e.g. kaons and protons, $\xi_{p}^{*}$ should be smaller than in the case of the $\pi^{0}$ distribution.

Comparisons of measured inclusive momentum spectra to the analytical QCD calculations have been published previously for charged particles $[3,7,16,17]$.

\section{The $\mathrm{L} 3$ detector}

The $\mathrm{L} 3$ detector covers $99 \%$ of $4 \pi$ [ 18 ]. The detector consists of a central tracking chamber, a high resolution electromagnetic calorimeter composed of bismuth germanium oxide crystals, a ring of scintil- lation counters, a uranium and brass hadron calorimeter with proportional wire chamber readout, and an accurate muon chamber system. These detectors are installed in a $12 \mathrm{~m}$ diameter magnet which provides a uniform field of $0.5 \mathrm{~T}$ along the beam direction.

The central tracking chamber is a time expansion chamber which consists of two cylindrical layers of 12 and 24 sectors, with 62 sense wires measuring the $R-\phi$ coordinate. The single wire resolution is $58 \mu \mathrm{m}$ averaged over the entire cell. The double-track resolution is $640 \mu \mathrm{m}$.

The material preceeding the electromagnetic detector amounts to less than $10 \%$ of a radiation length. The energy resolution is $5 \%$ for photons and electrons of energy around $100 \mathrm{MeV}$, and is less than $1 \%$ for energies above $\approx 2 \mathrm{GeV}$. The angular resolution of electromagnetic clusters is better than $0.5^{\circ}$ for energies above $1 \mathrm{GeV}$.

For the present analysis, we use the data collected in the following ranges of polar angles:

- for the central tracking chamber, $40^{\circ}<\theta<140^{\circ}$, - for the electromagnetic calorimeter, $42^{\circ}<\theta<138^{\circ}$, - for the hadron calorimeter, $5^{\circ}<\theta<175^{\circ}$.

\section{Selection of hadronic events}

Events collected at center of mass energies around $\sqrt{s}=91.2 \mathrm{GeV}(88.2 \leqslant \sqrt{s} \leqslant 94.2 \mathrm{GeV})$ from the 1990 LEP running period are used for this analysis.

The primary trigger for hadronic events requires a total energy of about $15 \mathrm{GeV}$ in the calorimeters. This trigger is in logical OR with a trigger using the barrel scintillation counters and with a charged track trigger. The combined trigger efficiency for selected hadronic events exceeds $99.9 \%$.

The selection of $\mathrm{e}^{+} \mathrm{e}^{-} \rightarrow$ hadrons events is based on the energy measured in the electromagnetic detector and in the hadron calorimeter. Events are accepted if

$$
\begin{aligned}
& 0.5<E_{\text {vis }} / \sqrt{s}<1.5, \\
& \left|E_{\|}\right| / E_{\text {vis }}<0.40, \quad E_{\perp} / E_{\text {vis }}<0.40, \\
& N_{\text {cluster }} \geqslant 15,
\end{aligned}
$$

where $E_{\text {vis }}$ is the total energy observed in the detector, $E_{\|}$is the energy imbalance along the beam direction, and $E_{\perp}$ is the transverse energy imbalance. An 
algorithm was used to group neighbouring calorimeter hits, which are probably produced by the same particle, into clusters. Only clusters with a total energy above $100 \mathrm{MeV}$ were used. The algorithm normally reconstructs one cluster for each particle produced near the interaction point. Thus the cut on the number of clusters rejects low multiplicity events $\left(\mathrm{e}^{+} \mathrm{e}^{-}, \mu^{+} \mu^{-}, \tau^{+} \tau^{-}\right)$.

In total 112000 events were selected.

Applying the same cuts to simulated events, we find that $97 \%$ of the hadronic decays from the $Z^{0}$ are accepted. The contamination from final states $\mathrm{e}^{+} \mathrm{e}^{-}$, $\tau^{+} \tau^{-}$and hadronic production via two-photon processes in the event sample is below $0.2 \%$ and can be neglected.

Monte Carlo events were generated by the parton shower program JETSET 7.2 [8] with values for the QCD scale and string fragmentation parameters as determined from a fit to our data $[19,20]$. The generated events were passed through the $\mathrm{L} 3$ detector simulation [21], which includes the effects of energy loss, multiple scattering, interactions and decays in the detector materials and beam pipe.

\section{Photon selection}

Photons are recognized as isolated and confined clusters in the electromagnetic calorimeter, a cluster being a matrix of $3 \times 3$ crystals centered on the most energetic crystal. The photon energy is calculated from the energy of the cluster by applying a positiondependent leakage correction. Assuming the photon to originate at the interaction point, the photon direction is determined from the geometrical positions of the constituent crystals, weighted by the corresponding energy deposits.

Clusters in the electromagnetic calorimeter are accepted as photons if the following requirements are met:

(1) $E_{\gamma}>130 \mathrm{MeV}$,

(2) $E_{\gamma} / E_{25}>0.9$,

(3) $E_{\text {had }} / E_{\gamma}<0.2$,

(4) $\theta_{\min }>100 \mathrm{mrad}$

where $E_{\gamma}$ denotes the photon energy and $E_{25}$ is the leakage corrected energy deposited in the $5 \times 5$ symmetric extension of the $3 \times 3$ crystal array. $E_{\text {had }}$ stands for the energy deposited in the six innermost hadron calorimeter layers inside a cone of half angle $100 \mathrm{mrad}$ around the photon direction. We define $\theta_{\min }$ as the angle between the direction of the photon candidate and the nearest cluster. Cuts (2) and (3) suppress background from hadrons. The lower bound of 100 mrad in (4) reflects the finite granularity of the electromagnetic calorimeter which limits the energy of $\pi^{0}$ mesons decaying into non-overlapping photons to about $3 \mathrm{GeV}$.

\section{Inclusive spectra for neutral pions}

The $\gamma \gamma$ invariant mass spectrum is measured using pairs of photons in an event in which both photons are in the same hemisphere defined by a plane perpendicular to the event thrust axis or if they belong to the same jet [22]. The invariant mass distribution of all photon pairs in the kinematic region $0.0075<x_{p}<0.065$, covered by the present analysis, is shown in fig. 1. The fit to the mass distribution, indicated by a solid line, is a sum of a gaussian function and a third order polynomial. The position of the $\pi^{0}$ signal peak is consistent with the neutral pion mass. It has a width of $\sigma=7.1 \pm 0.1 \mathrm{MeV}$, and contains $31300 \pm 400 \pi^{0}$ mesons. The observed resolu-

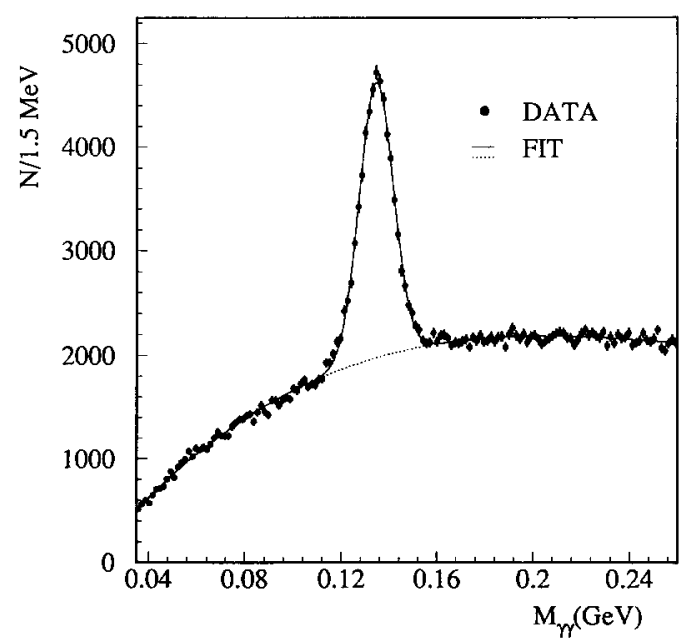

Fig. 1. Measured invariant mass distribution for pairs of photons. The solid line indicates the result of a fit to the data using the sum of a gaussian distribution and a third order polynomial. The dashed line indicates the background. The $\pi^{0}$ signal has a width of $\sigma=7.1 \pm 0.1 \mathrm{MeV}$ 
tion is consistent with the Monte Carlo expectations.

To determine the $x_{p}$ distribution of reconstructed $\pi^{0}$ mesons, the measurement of the invariant mass distribution and the fit are repeated for different $x_{p}$ intervals. The $\pi^{0}$ yield as a function of the variable $\xi_{p}$ is obtained in a similar way. The widths of the $x_{p}$ and $\xi_{p}$ bins are chosen such that the bin-to-bin migration, as determined from Monte Carlo studied, is small. In the case of the $x_{p}\left(\xi_{p}\right)$ distribution, this smearing effect is of the order of $20 \%(10 \%)$.

To measure the differential cross sections the observed yields of $\pi^{0}$ mesons in the data are corrected, bin by bin, for detector effects (acceptance, efficiency and resolution). The detector correction factors are calculated using the JETSET Monte Carlo events that are passed through the detector simulation and reconstruction programs. The $\pi^{0}$ detection efficiencies are found to be $\approx 6 \%$ in the center of the kinematic range and approach $2 \%$ at both ends. The JETSET program is also used to compute the correction factors for initial and final state photon radiation, which are equal to one within $1 \%$.

Systematic uncertainties in the detector correction are estimated from the difference in the corrected spectra obtained with two different sets of detector calibration constants. In addition, we replaced the fitted JETSET fragmentation parameters by the corresponding default values and determined the resulting detection efficiencies. The different contributions to the systematic errors, of which the fragmentation uncertainty is the dominant one, are added in quadrature. The systematic errors for adjacent $x_{p}\left(\xi_{p}\right)$ intervals are correlated.

The differential cross sections for inclusive $\pi^{0}$ production at the $Z^{0}$ resonance, normalized to the total hadronic cross section, $\sigma_{\mathrm{h}}$, are shown in table 1 and figs. $3 a$ and $4 a$ as a function of the variables $x_{p}$ and $\xi_{p}$, respectively.

Integrating over the interval $0.0075<x_{p}<0.065$, we find an average $\pi^{0}$ multiplicity of $\left\langle\tilde{n}_{\pi^{0}}\right\rangle=6.4 \pm 0.1$ (stat.) \pm 0.4 (syst.). The corresponding numbers predicted by the Monte Carlo programs JETSET and HERWIG are $\left\langle\tilde{n}_{\pi 0}\right\rangle=6.3$ and 6.4 , respectively. The predictions for the total number of $\pi^{0}$ particles per event are $\left\langle n_{\pi^{0}}\right\rangle=9.7$ and 9.9 , respectively.
Table 1

Differential cross sections for inclusive $\pi^{0}$ production, normalized to the total hadronic cross section at the $Z^{0}$ peak. The errors given are statistical and systematic. The statistical errors include the statistical uncertainties of the detector correction factors calculated using Monte Carlo events. The systematic errors for adjacent $x_{p}\left(\xi_{p}\right)$ intervals are correlated.

\begin{tabular}{lrll}
\hline$x_{p}$ & $\left(1 / \sigma_{\mathrm{h}}\right) \mathrm{d} \sigma / \mathrm{d} x_{p}$ & $\xi_{p}$ & $\left(1 / \sigma_{\mathrm{h}}\right) \mathrm{d} \sigma / \mathrm{d} \xi_{p}$ \\
\hline $0.0075-0.010$ & $304 \pm 35 \pm 20$ & $2.8-3.0$ & $1.79 \pm 0.11 \pm 0.70$ \\
$0.010-0.015$ & $283 \pm 16 \pm 30$ & $3.0-3.2$ & $2.22 \pm 0.10 \pm 0.50$ \\
$0.015-0.020$ & $207 \pm 10 \pm 20$ & $3.2-3.4$ & $2.85 \pm 0.12 \pm 0.30$ \\
$0.020-0.025$ & $153 \pm 7 \pm 15$ & $3.4-3.6$ & $3.14 \pm 0.13 \pm 0.30$ \\
$0.025-0.030$ & $121 \pm 6 \pm 10$ & $3.6-3.8$ & $3.40 \pm 0.16 \pm 0.30$ \\
$0.030-0.035$ & $94 \pm 5 \pm 10$ & $3.8-4.0$ & $3.44 \pm 0.17 \pm 0.30$ \\
$0.035-0.040$ & $74 \pm 4 \pm 10$ & $4.0-4.2$ & $3.64 \pm 0.21 \pm 0.30$ \\
$0.040-0.045$ & $54 \pm 3 \pm 10$ & $4.2-4.4$ & $3.85 \pm 0.27 \pm 0.30$ \\
$0.045-0.050$ & $46 \pm 3 \pm 10$ & $4.4-4.6$ & $3.18 \pm 0.28 \pm 0.30$ \\
$0.050-0.055$ & $39 \pm 3 \pm 10$ & $4.6-4.8$ & $2.75 \pm 0.25 \pm 0.30$ \\
$0.055-0.060$ & $31 \pm 3 \pm 9$ & $4.8-5.0$ & $3.15 \pm 0.60 \pm 0.30$ \\
$0.060-0.065$ & $24 \pm 4 \pm 7$ & & \\
\hline
\end{tabular}

\section{Inclusive spectra for charged particles}

Charged tracks are reconstructed in the central tracking chamber, which measures the momentum transverse to the beam axis. The $z$ coordinate is determined from the position of the cluster closest to the track in the plane perpendicular to the beam axis.

To determine the inclusive momentum distribution for charged particles, we analyze 73000 hadronic events at $\sqrt{s}=91.2 \mathrm{GeV}$. Only those events with $|\cos \theta|<0.6$ are used, where $\theta$ is the angle of the thrust axis with respect to the beam line. Tracks are selected if

$-N_{\text {hits }}>35$,

$-d_{\perp}<5 \mathrm{~mm}$,

$-p_{\perp}>100 \mathrm{MeV}$,

where $N_{\text {hits }}$ denotes the number of measured points on the track, $d_{\perp}$ is the distance of closest approach to the beam axis in the transverse plane, and $p_{\perp}$ is the transverse momentum with respect to the beam axis.

Fig. 2 shows the measured corrected charged multiplicity distribution. The data have been corrected for detector acceptance, resolution, reconstruction efficiency, and photon radiation. The unfolding is based on a matrix relating the Monte Carlo generated charged multiplicity distribution to the one obtained from the corresponding simulated and reconstructed 


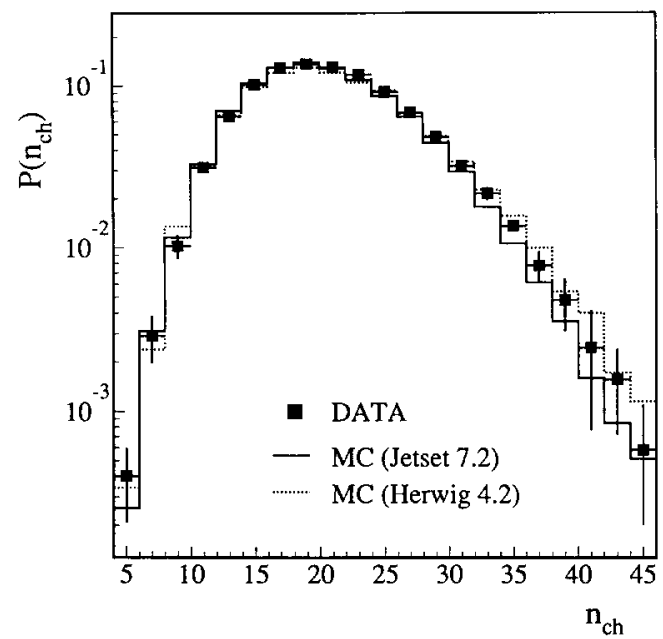

Fig. 2. Multiplicity distribution for charged particles in comparison with the predictions of the Monte Carlo programs JETSET 7.2 and HERWIG 4.2. $P\left(n_{\mathrm{ch}}\right)$ denotes the probability for finding $n_{\mathrm{ch}}$ charged particles in a hadronic $Z^{0}$ decay. The errors (vertical bars) include the statistical and systematic uncertainties. The horizontal bars indicate the bin size. The average charged multiplicity is $\left\langle n_{\mathrm{ch}}\right\rangle=20.7 \pm 0.7$.

events. For the generated events, all the primary produced particles or those produced in the decay of particles with an average lifetime smaller than $5 \times 10^{-10} \mathrm{~s}$ are considered. The measured average multiplicity is $\left\langle n_{\mathrm{ch}}\right\rangle=20.7 \pm 0.7$. The error is dominated by systematic uncertainties, which are estimated by using two different Monte Carlo generators (JETSET and HERWIG) for the unfolding procedure, and by a variation of track selection parameters. The corresponding numbers predicted by the Monte Carlo programs JETSET and HERWIG are $\left\langle n_{\mathrm{ch}}\right\rangle=20.5$ and 20.8 , respectively. Our results are in agreement with other measurements at the $Z^{0}$ peak [23].

The measured charged particle $x_{p}$ and $\xi_{p}$ distributions are corrected, bin by bin, for acceptance, reconstruction efficiency and resolution. The bin width is chosen to be bigger than the resolution, so that the bin-to-bin migration is small. This leads to 12 bins for the $x_{p}$ spectrum in the range $0.002 \leqslant x_{p} \leqslant 0.1$, and 12 bins for the $\xi_{p}$ distribution in the range $1.1 \leqslant \xi_{p} \leqslant 5.4$. To determine the detector correction factors, we use the same Monte Carlo events as for the $\pi^{0}$ analysis. The factors deviate from one by less than $5 \%$. The corrections factors for photon radia-
Table 2

Differential cross sections for inclusive charged particle production normalized to the total hadronic cross section at $\sqrt{s}=91.2$ $\mathrm{GeV}$. The errors include statistical and bin to bin systematic uncertainties. An additional uncertainty of $3 \%$ common to all bins is not shown.

\begin{tabular}{llll}
\hline$x_{p}$ & $\left(1 / \sigma_{\mathrm{h}}\right) \mathrm{d} \sigma / \mathrm{d} x_{p}$ & $\xi_{p}$ & $\left(1 / \sigma_{\mathrm{h}}\right) \mathrm{d} \sigma / \mathrm{d} \xi_{p}$ \\
\hline $0.002-0.006$ & $370 \pm 4$ & $1.1-1.6$ & $1.62 \pm 0.07$ \\
$0.006-0.010$ & $502 \pm 4$ & $1.6-2.1$ & $3.00 \pm 0.09$ \\
$0.010-0.014$ & $453 \pm 5$ & $2.1-2.5$ & $4.31 \pm 0.08$ \\
$0.014-0.018$ & $375 \pm 5$ & $2.5-2.9$ & $5.34 \pm 0.10$ \\
$0.018-0.022$ & $315 \pm 5$ & $2.9-3.3$ & $6.00 \pm 0.08$ \\
$0.022-0.030$ & $248 \pm 5$ & $3.3-3.6$ & $6.39 \pm 0.10$ \\
$0.030-0.040$ & $181 \pm 4$ & $3.6-3.9$ & $6.35 \pm 0.12$ \\
$0.040-0.050$ & $135 \pm 3$ & $3.9-4.2$ & $6.09 \pm 0.12$ \\
$0.050-0.060$ & $104 \pm 3$ & $4.2-4.5$ & $5.61 \pm 0.12$ \\
$0.060-0.070$ & $83 \pm 3$ & $4.5-4.8$ & $4.71 \pm 0.09$ \\
$0.070-0.085$ & $66 \pm 3$ & $4.8-5.1$ & $3.61 \pm 0.06$ \\
$0.085-0.100$ & $49 \pm 3$ & $5.1-5.4$ & $2.49 \pm 0.05$
\end{tabular}

tion are equal to one within $1 \%$.

Systematic errors are estimated by changing the fragmentation parameters in the JETSET Monte Carlo within their errors [19] and by varying the parameters in the simulation of the tracking chamber. The relative systematic bin-to-bin error varies between $1 \%$ and $5 \%$. Statistical uncertainties are negligible. An additional uncertainty of $3 \%$ related to the tracking chamber reconstruction efficiency is common to all bins.

The differential cross sections for inclusive charged particle production at the $Z^{0}$ resonance, normalized to the total hadronic cross section, are shown in table 2 and figs. $3 b$ and $4 b$.

\section{Comparison to $\mathrm{QCD}$ predictions}

In fig. 3 the measured cross sections $\left(1 / \sigma_{\mathrm{h}}\right) \mathrm{d} \sigma / \mathrm{d} x_{p}$ for neutral pions and charged particles are compared to the predictions of the parton shower Monte Carlo programs JETSET and HERWIG, which reproduce the data. The QCD scale and fragmentation parameters used in the Monte Carlo programs are determined from a comparison to our measured hadronic event shape distributions and inclusive muon spectra [19,20].

Fig. 4 shows our measurements of the cross sec- 

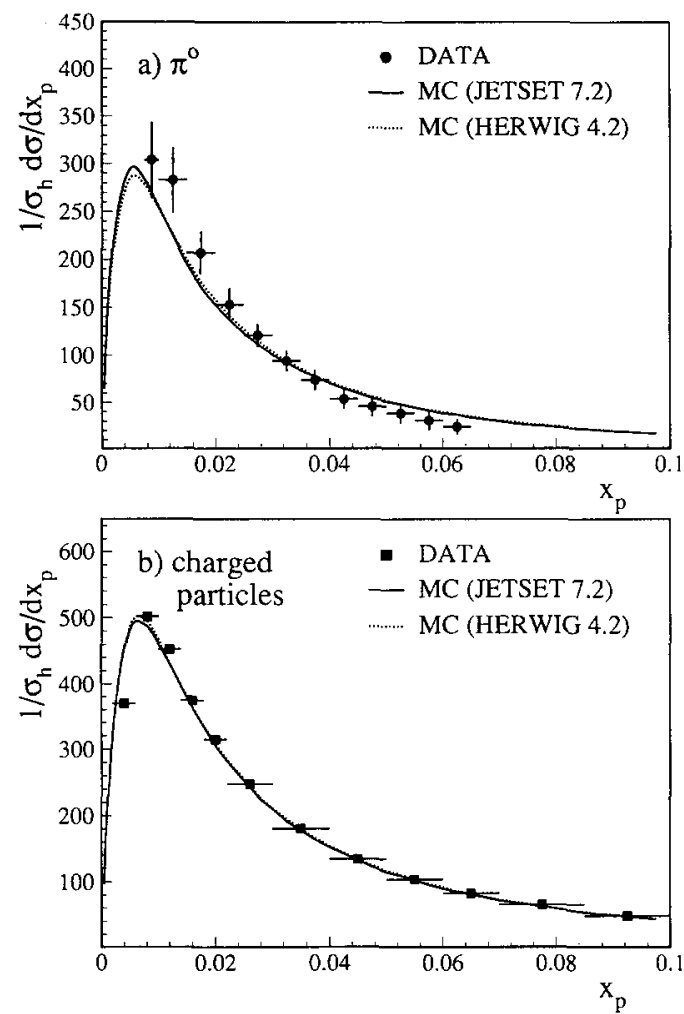

Fig. 3. The $x_{p}$ spectrum for inclusive particle production at the $\mathrm{Z}^{0}$ peak normalized to the total hadronic cross section in comparison with parton shower Monte Carlo predictions for (a) neutral pions and (b) charged particles. The errors (vertical bars) include statistical and systematic uncertainties. The uncertainty of $3 \%$ common to all bins of the charged particle spectrum is not shown. The horizontal bars indicate the bin size.

tions $\left(1 / \sigma_{\mathrm{h}}\right) \mathrm{d} \sigma / \mathrm{d} \xi_{p}$, as well as spectra measured at lower center of mass energies $[2,5,6]$. The data are compared to the predictions of the MLLA QCD calculations [10], which can be written in the form

$\frac{1}{\sigma_{\mathrm{h}}} \frac{\mathrm{d} \sigma}{\mathrm{d} \xi_{p}}=N(\sqrt{s}) f\left(\sqrt{s}, \Lambda_{\mathrm{eff}} ; \xi_{p}\right)$,

where $\Lambda_{\text {eff }}$ is an effective scale parameter, which is not directly related to $\Lambda_{\overline{\mathrm{MS}}} . f$ is a function of $\sqrt{s}, \Lambda_{\mathrm{eff}}$ and $\xi_{p}$. An increase in $A_{\text {eff }}$ corresponds to a decrease in the position of the maximum, $\xi_{p}^{*}$. The normalization factor $N$, which describes the hadronization, is a function of the center of mass energy $\sqrt{s}$ and the particle type. The number of colours and light flavours are
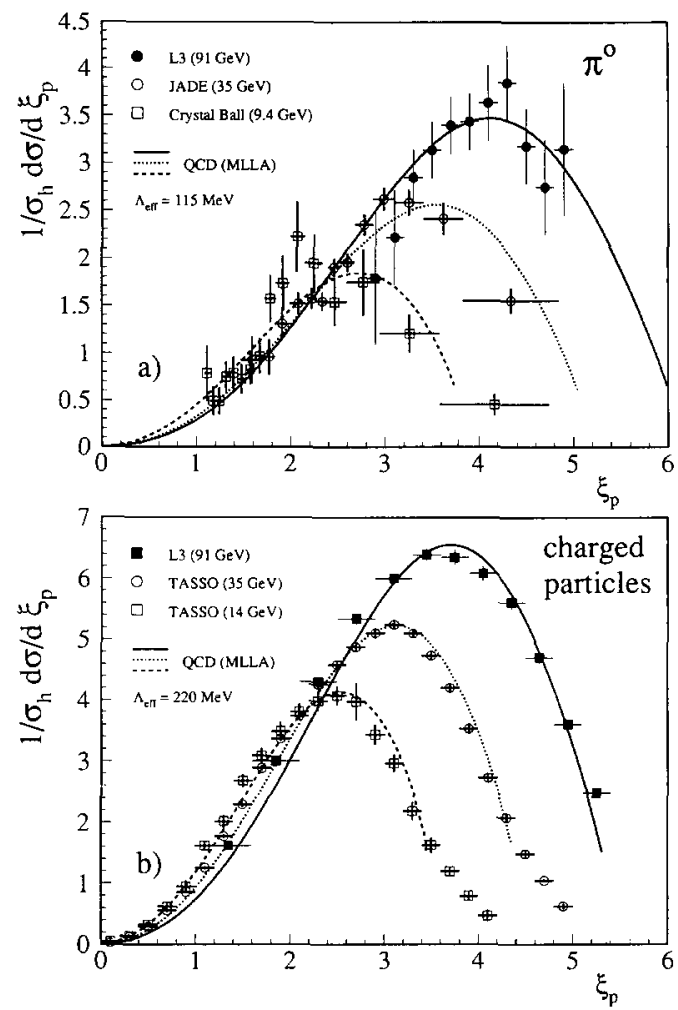

Fig. 4. Inclusive $\xi_{p}$ spectrum normalized to the total hadronic cross section in comparison with the analytical QCD calculations for (a) neutral pions and (b) charged particles. Besides the L3 spectra, results from the Crystal Ball, JADE and TASSO collaborations are shown. The errors include statistical and bin-to-bin systematic uncertainties. The value of $A_{\text {eff }}$ used in the QCD calculation is determined from a fit to the L3 data. The horizontal bars indicate the bin size.

both set to 3 when calculating $f$. Eq. (1) is defined in the range $0<\xi_{p}<\ln \left(0.5 \sqrt{s} / A_{\text {eff }}\right)$.

We fit expression (1) to our data in a range of $\xi_{p}$ of $\approx \pm 1$ around the position $\xi_{p}^{*}$ of the maximum, with the only free parameters being $\Lambda_{\text {eff }}$ and $N$. We obtain

$\pi^{0}: A_{\mathrm{eff}}=115 \pm 32 \pm 20 \mathrm{MeV}$,

$$
\xi_{p}^{*}=4.11 \pm 0.15 \pm 0.10,
$$

charged particles: $A_{\mathrm{eff}}=220 \pm 3 \pm 20 \mathrm{MeV}$,

$\xi_{p}^{*}=3.71 \pm 0.01 \pm 0.05$.

$\xi_{p}^{*}$ is the position of the maximum as calculated from (1). The first error on each parameter is the uncer- 
tainty corresponding to the combined statistical and systematic errors of the data points used in the fit. The second error is systematic and is estimated from a variation of the fit range by up to two bins at both the lower and upper end. The normalization factors are

$\pi^{0}: N=0.52 \pm 0.05$,

charged particles: $N=1.20 \pm 0.04$.

The QCD predictions for $\sqrt{s}=91 \mathrm{GeV}$ based on the fitted parameters (2) $-(5)$ are shown in fig. 4 as solid lines. The QCD spectra for lower center of mass energies are obtained from a fit to the corresponding data using the same value of $A_{\text {eff }}$ as determined from our data at $91 \mathrm{GeV}$, with the normalization constant $N$ as the only free parameter. Around $\xi_{p}^{*}$ the MLLA calculations reproduce all measurements in the wide center of mass energy range $9-91 \mathrm{GeV}$ with the same effective scale $A_{\text {eff }}$. There is agreement also for small values of $\xi_{p}$, while for very low particle momenta the cross sections cannot be calculated. The value of $\Lambda_{\text {eff }}$ obtained for charged particles is higher than the value for neutral pions, as predicted.

Since the QCD calculations are performed for massless partons, they cannot distinguish momentum $p$ from energy $E$. Therefore we have also compared the QCD predictions to our measured $\xi_{E}=\ln \left(1 / x_{E}\right)=\ln (0.5 \sqrt{s} / E)$ distributions. In the case of the charged particles, we have used the charged pion mass to calculate the energy $E$. The fitted $\Lambda_{\text {eff }}$ values are shifted with respect to those in (2) and (3) by -20 and $-10 \mathrm{MeV}$, respectively.

In order to study in detail the energy dependence of the inclusive $\pi^{0}$ and charged particle $\xi_{p}$ distributions, we determined for all the available spectra between 9 and $91 \mathrm{GeV}$ which cover the peak region [17] the parameters $A_{\text {eff }}$ and $N$ by fitting the MLLA function (1) as described above for our data. The corresponding peak positions $\xi_{p}^{*}$ are shown in fig. 5 . Calculating the weighted mean values of $A_{\text {eff }}$ for all neutral pion data and for all charged particle spectra gives

$\pi^{0}: A_{\mathrm{eff}}=160 \pm 15 \mathrm{MeV}$,

charged particles: $\Lambda_{\mathrm{eff}}=240 \pm 15 \mathrm{MeV}$.

The errors include uncertainties related to the choice of fit range. The evolution of the peak position is con-
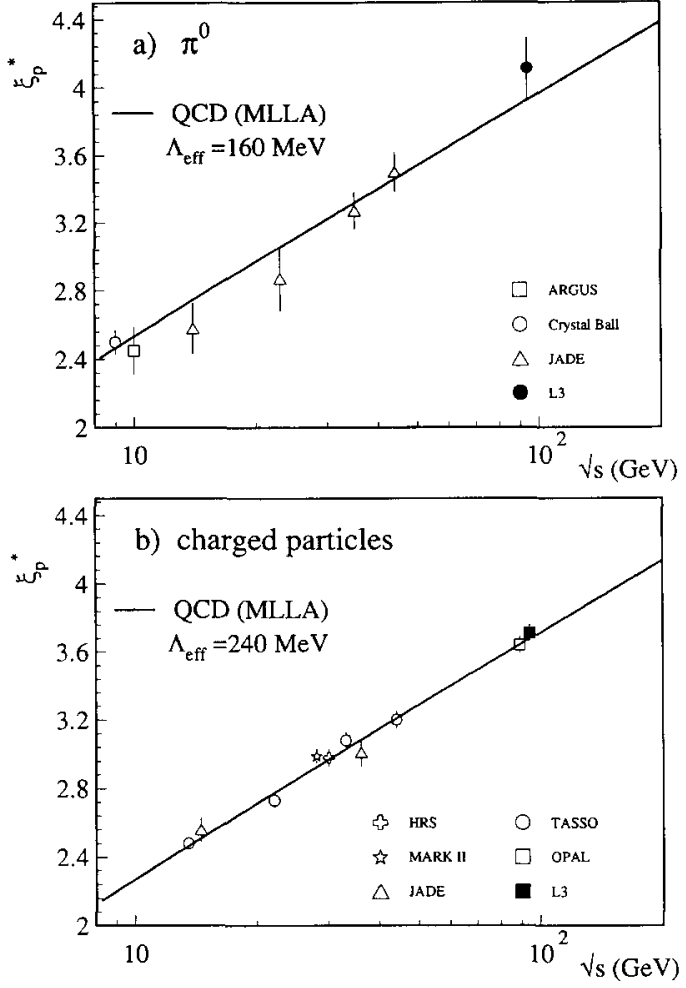

Fig. 5. Energy dependence of the position of the maximum, $\xi_{p}^{*}$, in the measured $\xi_{p}$ distributions for (a) neutral pions and (b) charged particles. The maximum is determined from a fit to the measured spectra using the MLLA calculations. The errors include statistical uncertainties as well as systematic effects estimated from a variation in the fit range. The lines are the QCD predictions using values for $A_{\text {eff }}$ determined from a fit to all points in (a) or (b). For a better readability of this graph, different points at the same center of mass energy were slightly shifted horizontally.

sistent with the QCD formula (1), as can be seen from fig. 5 .

\section{Summary and conclusions}

We have measured the production of charged particles and neutral pions from 112000 hadronic $\mathrm{Z}^{\circ}$ decays. This is the first study of the inclusive production of identified hadrons at the $Z^{0}$ resonance. The measured inclusive momentum distributions can be reproduced by parton shower Monte Carlo programs and also by an analytical QCD calculation. We find effective scale parameters 
$\Lambda_{\mathrm{eff}}=115 \pm 38 \mathrm{MeV}\left(\pi^{0}\right)$,

$A_{\text {eff }}=220 \pm 20 \mathrm{MeV}$ (charged particles) .

Comparing our results to $\mathrm{e}^{+} \mathrm{e}^{-}$data between $\sqrt{s}=9$ and $91 \mathrm{GeV}$, we find that the center of mass energy evolution of the spectra is consistent with the QCD predictions.

\section{Acknowledgement}

We wish to express our gratitute to the CERN accelerator divisions for the excellent performance of the LEP machine. We acknowledge the effort of all engineers and technicians who have participated in the construction and maintenance of this experiment.

\section{References}

[1] ARGUS Collab., H. Albrecht et al., Z. Phys. C 46 (1990) 15.

[2] CRYSTAL BALL Collab., C. Bieler et al., DESY Report 90086 (1990).

[3] HRS Collab., D. Bender et al., Phys. Rev. D 31 (1985) 1.

[4] MARK II Collab., J.F. Patrick et al., Phys. Rev. Lett. 49 (1982) 1232

[5] JADE Collab., W. Bartel et al., Z. Phys. C 20 (1983) 187; C 28 (1985) 343; C 46 (1990) 1.

[6] TASSO Collab., W. Braunschweig et al., Z. Phys. C 33 (1986) 13; C 42 (1989) 189; C 47 (1990) 187.

[7] OPAL Collab., M.Z. Akrawy et al., Phys. Lett. B 247 (1990) 617.

[8] T. Sjöstrand, Comput. Phys. Commun. 39 (1986) 347; T. Sjöstrand and M. Bengtsson, Comput. Phys. Commun. 43 (1987) 367

[9] G. Marchesini and B. Webber, Nucl. Phys. B 310 (1988) 461.

[10] Y.L. Dokshitzer and S.I. Troyan, Leningrad preprint LNPI922 (1984);
Y.I. Azimov et al., Z. Phys. C 27 (1985) 65; C 31 (1986) 213;

V.A. Khoze, Y.L. Dokshitzer and S.I. Troyan, Lund preprint LU-TP 90-12.

[11] D. Amati and G. Veneziano, Phys. Lett. B 83 (1979) 87.

[12] For an introduction see for example Z. Kunszt and P. Nason, Z. physics at LEP 1, CERN report CERN-89-08, Vol. I., p. 373

[13] A.H. Mueller, Phys. Lett. B 104 ( 1981 ) 161;

A. Bassetto et al., Nucl. Phys. B 207 (1982) 189;

B.I. Ermolaev and V.S. Fadin, JETP Lett. 33 (1981) 285;

Y.L. Dokshitzer, V.S. Fadin and V.A. Khoze, Phys. Lett. B 115 (1982) 242.

[14] A.H. Mueller, Nucl. Phys. B 213 (1983) 85; B 241 (1984) $141(\mathrm{E})$.

[15] C.P. Fong and B.R. Webber, Phys. Lett. B 22991989$) 289$.

[16] TPC Collab., H. Aihara et al., Phys. Rev. Lett. 52 (1984) 577.

[17] TASSO Collab., M. Althoff et al., Z. Phys. C 22 (1984) 307.

[18] L3 Collab., B. Adeva et al., Nucl. Instrum. Methods A 289 (1990) 35.

[19] L3 Collab., B. Adeva et al., Phys. Lett. B 257 (1991) 469.

[20] L3 Collab., B. Adeva et al., Phys. Lett. B 241 (1990) 416;

V. Innocente (L3 Collab.), A measurement of the $\mathrm{Z}^{0} \rightarrow \mathrm{b} \overline{\mathrm{b}}$ partial decay width, talk at 25 th Intern. Conf. on High energy physics (Singapore, 1990).

[21] GEANT Version 3.13 (September, 1989), see R. Brun et al., GEANT 3, CERN DD/EE/84-1 (revised) (September 1987);

to simulate hadronic interactions the program GHEISHA is used, see H. Fesefeldt, RWTH Aachen preprint PITHA $85 / 02(1985)$

[22] O. Adriani et al., CERN preprint CERN-PPE/90-158.

[23] MARK II Collab., G.S. Abrams et al., Phys. Rev. Lett. 64 (1990) 1334;

ALEPH Collab., D. Decamp et al., Phys. Lett. B 234 (1990) 209;

DELPHI Collab., P. Aarnio et al., Phys. Lett. B 240 (1990) 271 ;

DELPHI Collab., P. Abreu et al., CERN preprint CERNPPE/90-173;

OPAL Collab., M.Z. Akrawy et al., Z. Phys. C 47 (1990) 505. 